\title{
Memory strength versus memory variability in visual change detection
}

\author{
Robert M. Nosofsky ${ }^{1,2} \cdot$ Jason Gold $^{1}$
}

Published online: 20 October 2015

(C) The Psychonomic Society, Inc. 2015

\begin{abstract}
Observers made change-detection judgments for colored squares in a paradigm that manipulated the retention interval, the magnitude of change, and objective change probability. The probability of change judgments increased across the retention interval for "same" and "small-change" test items but stayed the same or decreased for "large-change" and "far" test items. A variety of formal models were fitted to the individual-subject data. The modeling results provided evidence that, beyond changes in visual-memory precision, there were decreases in memory strength of individual study items across the retention interval. In addition, the modeling results provided evidence of a zero-information, absence-of-memory state that required guessing. The data were not sufficiently strong to sharply distinguish whether the losses in memory strength across the retention interval were continuous in nature or all-or-none. The authors argue that the construct of memory strength as distinct from memory variability is an important component of the nature of forgetting from visual working memory.
\end{abstract}

Keywords Visual change detection · Memory models · Memory strength $\cdot$ Visual memory

In this research we examined the issue of how visual working memories (VWMs) are lost over time. The predominant view in the field is that perceptual memories become progressively less precise with increases in the retention interval, with

Robert M. Nosofsky

nosofsky@indiana.edu

1 Indiana University Bloomington, Bloomington, IN, USA

2 Psychological and Brain Sciences, Indiana University, 1101 E. Tenth Street, Bloomington, IN 47405, USA distinct visual objects becoming gradually less discriminable from one another (e.g., Magnussen \& Greenlee, 1999). Classic work has examined alternative psychophysical functions on their ability to describe the relation between perceptual discriminability and the retention interval (for a review and critical analysis, see, e.g., Laming \& Scheiwiller, 1985). Models based on diffusion processes have been developed to provide mechanistic explanations for these gradual losses in precision and discriminability (e.g., Kinchla \& Smyzer, 1967).

Modern work in visual working memory has also examined the ability of precision-based models to explain declines in perceptual discriminability that arise with increases in the retention interval (e.g., Bays, Catalao, \& Husain, 2009; Brady, Konkle, Gill, Oliva, \& Alvarez, 2013). Some of the main metaphors in these forms of modeling are that perceptual representations become increasingly "fuzzy" or more variable as the retention interval increases. In this modern work, the main procedure used for examining these issues has been the continuous-recall paradigm (Wilken \& Ma, 2004; Zhang \& Luck, 2008). In this paradigm, observers view a set of study objects, such as colors in different locations that vary in their hue. Following a variable retention interval, a single location is probed, and the observer is required to reproduce the color that existed at that location by clicking on the appropriate portion of a color-wheel response device. According to precision-loss theories, the perceptual memory of the studied color follows a bell-shaped probability distribution that becomes more variable with the passage of time. Such models have yielded excellent quantitative accounts of performance in the color-reproduction task.

The central thesis that we advance in the present work is that beyond changes in visual-memory precision or variability, there are decreases in memory strength as the retention interval increases. We formally distinguish between precision and strength in the context of our subsequent model-fitting 
analyses. In brief, and as we expand upon below, whereas precision influences the extent to which distinct objects are similar to one another, strength is a construct pertaining to the memory representation of a single object. We pursue this thesis involving the joint roles of precision and strength in VWM forgetting by examining performance in a changedetection paradigm rather than the continuous-recall task (e.g., Luck \& Vogel, 1997; Pashler, 1988; Rouder et al., 2008). In the present change-detection paradigm, a single location from the visual display was probed with a color, and the observer was required to judge whether the color at that location changed or stayed the same. As we explain below, the change-detection paradigm allowed us to test for key aspects of the nature of VWM forgetting, about which the continuousrecall paradigm does not provide information.

\section{Variability versus strength}

We had three main motivations for testing the changedetection version of the task. The primary motivation was that we sought to gain evidence of more detailed forms of memory loss than is currently hypothesized in extant precision-based accounts of VWM. As noted earlier, the dominant approach to modeling precision loss in VWM is in terms of increased variance of an underlying perceptual memory distribution. Thus, in a color-recall task, the locations to which the observer points on the color wheel will become increasingly variable. Alternatively, in making change/same judgments, the ability to discriminate between distinct visual objects may decrease.

In our view, however, the hypothesis that the value of the remembered color simply becomes more variable with the passage of time is only part of what may constitute visual memory loss. A conceptually distinct idea is that the strength of the memory trace of the to-be-remembered object may also decrease with the passage of time. Ultimately, we will flesh out these constructs in terms of distinct formal parameters of mathematical models of VWM change detection. We start, however, by trying to provide intuitions of the psychological meaning of the terms.

Suppose that an observer studies the color green. Following a retention interval, the memory for the color may wander from the true value, such that the remembered value is now closer to aqua. Although the remembered value of the object has undergone change, something more seems to be involved in fully characterizing the nature of the memory loss. Subjectively, besides changing value, the original memory seems also to have faded away. Stated another way, the retained memory seems to have less intensity than immediately after the original study experience. The memory-strength construct that we incorporate in our modeling is aimed at formalizing this intuition.
Indeed, numerous memory models outside the domain of VWM have distinct formal constructs related to similarity (discriminability between pairs of objects) and strength (a factor pertaining to individual objects). For example, in spreading-activation models of memory (e.g., Anderson, 1990), individual items are represented as nodes and relations between items are represented as links. Items that are more closely related are connected by stronger links. In addition, however, the individual-item nodes may have different baseline strengths determined by factors such as the frequency or recency with which those individual items have been experienced. The extent to which an individual-item node is activated is determined jointly by its baseline strength in memory and the spreading activation it receives from its connecting links.

Likewise, formal exemplar models of perceptual identification and categorization and short-term and long-term memory have long distinguished between the similarity between distinct exemplars and the memory-strength or stimulus bias associated with those individual exemplars (e.g., Kahana \& Sekuler, 2002; Luce, 1963; Nosofsky, 1991a, 1991b; Nosofsky, Little, Donkin, \& Fific, 2011; Shepard, 1957). Upon presentation of a test probe, the extent to which any exemplar is activated is a joint function of its similarity to the test probe and its underlying memory strength. For example, in recent work, Nosofsky et al. (2011; Nosofsky, Cox, Cao, \& Shiffrin, 2014) used an exemplar-model approach to accounting for performance in visual short-term memory-search tasks. In these tasks, the observer is presented with a short list of study items, followed by a test probe, and the observer judges whether the test probe occurred in the study list. Nosofsky et al. (2011; Nosofsky et al., 2014) found that accuracies and response times (RTs) in the task were dramatically influenced by the recency with which a test probe had been presented on the study list, with more recently presented items giving rise to more accurate responding and faster RTs. The results were well modeled by assuming that, independent of the test probe that was presented, the strength of the individual study items on the list decreased as their lag of presentation increased.

By analogy, in a VWM change-detection task, it seems reasonable to hypothesize that the probability that the observer judges a test probe to be the same as a remembered object may be influenced by two factors: (a) the similarity between the test probe value and the remembered value, which will be influenced by the amount of memory variability that has taken place, and (b) the strength of the remembered value. In a continuous-recall color-reproduction task, one is attempting to probe only the estimated value of the remembered object: the strength of that remembered value is not being assessed. As will be seen, in the present study, by testing instead how changedetection judgments vary with the retention interval, we sought to test for a joint role of variability and strength in VWM. 


\section{Gradual decay versus sudden death}

Our second motivation for conducting the change-detection paradigm was to possibly obtain a more sensitive test of whether declines in VWM performance operate according to principles of gradual decay in precision and strength or according to "sudden death." In an influential study, Zhang and Luck (2009) provided evidence that rather than undergoing gradual declines in precision with increases in the retention interval, visual working memories undergo sudden death: at some particular moment in time, there is a complete loss of the visual memory representation (see also Donkin, Nosofsky, Gold, \& Shiffrin, 2015). In cases of sudden death, the observer is forced to guess regarding the identity of a presented study item. The sudden-death hypothesis can be viewed as providing an extreme form of the loss-of-memory-strength hypothesis: at some moment in time, there is a complete absence of memory for the study item (i.e., zero strength).

The evidence for sudden death, however, has been obtained using the continuous-recall paradigm. A potential limitation is that the properties of the continuous-recall paradigm itself may interfere with the information stored in VWM. For example, in the typical version of the paradigm, the response is produced by indicating a location along a continuous-valued response device. Note that presentation of the device itself (in which all continuous values are simultaneously present) may be highly interfering of the original memory, thereby leading to underestimates of the amount of information that was immediately available when memory was probed (e.g., Souza, Rerko, Lin, \& Oberauer, 2014). Because the change-detection paradigm involves presentation of only a single-valued test probe in a given location, it might not lead to the same pronounced interference, so could perhaps provide a more sensitive test of a role of gradual and fine-grained visual-memory decay.

In an effort to develop diagnostic tests between gradualdecay and sudden-death explanations, we manipulated in our design the magnitude of change on change trials. First, the magnitude of change was either small or big. As explained in our Modeling Analyses section, the big-change trials were included because they could help to provide evidence of sudden-death and guessing processes. Second, on smallchange trials, we manipulated the distance of the test probe from the to-be-remembered color (1, 2, or 3 distance units). Our hope was that this latter manipulation might provide evidence of a gradual-decay process (perhaps operating alongside sudden-death mechanisms). The intuition is illustrated in Fig. 1, which shows how confusability (or similarity) between colors is presumed to vary with psychological distance and the precision of memory. Following classic work (Shepard, 1987), our modeling presumes that similarity is an exponential decay function of psychological distance, with higher precision memory corresponding to a steeper slope of the

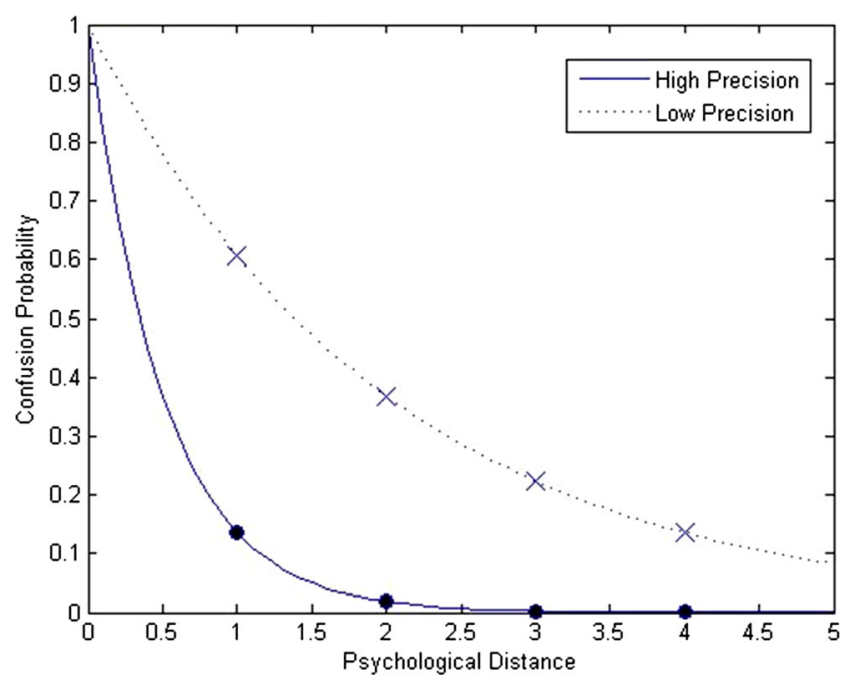

Fig. 1 Schematic illustration of how confusion probability (or "similarity") varies with psychological distance and memory precision (sensitivity)

exponential function. If there is decay in precision with time, then the increase in confusion probabilities as one shifts from the high-precision to the low-precision curve is predicted to be nonuniform across distances 1 through 3 . By contrast, if only sudden death operates, then the increase in confusion probabilities is predicted to be more nearly uniform.

\section{Sudden death versus extreme variability}

The third motivation for our study was to conduct preliminary tests of a potential variable-resources account of the manner in which VWM declines with the retention interval (e.g., Fougnie, Suchow, \& Alvarez, 2012; Sims, Jacobs, \& Knill, 2012; van den Berg, Shin, Chou, George, \& Ma, 2012). Variable-resources models have been applied successfully to account for the well-known finding that VWM performance declines with increases in memory set size. Such models adopt the idea that memory representations are doubly stochastic: The hypothesis is that not only does the variability of the memory representations tend to increase as set size increases but, in addition, there is a great deal of variability across individual items in terms of how variable these memory representations are. In an extreme case, for example, because minimal memory resources may be devoted to some particular item, an extremely dispersed memory representation may develop for that item. If an observer were relying on this type of extremely dispersed representation to recall a color in the continuous-recall task, it would be akin to a random guessing process. Indeed, van den Berg, Awh, and Ma (2014) found that such variable-resources models provided better detailed quantitative fits to continuous-recall data than did models that assumed mixtures of perceptual memory and guessing. 
Variable-resources models might be adapted to account for how VWM declines with the retention interval by positing that there is some probability that a highly dispersed memory representation develops for items at some point in time. Note that whereas the sudden-death hypothesis posits the absence of memory (and so the need for a true guessing process), the variable-resources model instead posits the presence of a highly variable representation. In our view, these hypotheses are conceptually and psychologically distinct. Furthermore, as will be seen, the hypotheses can be distinguished with use of the present VWM change-detection task.

To anticipate the results our study, we believe that we were successful in achieving some but not all of these goals. Specifically, we believe that our results provide clear evidence that change-detection judgments across the retention interval involve something more than only increased perceptualmemory variability or confusability. Something akin to changes in individual-item memory strength (or related item biases) also appear to play a major role. Furthermore, we find evidence for the role of a zero-information state involving the absence of memory in the paradigm: The alternative hypothesis of extreme variability from variable-resources models does not appear to be a substitute for the absence-of-memory construct. Despite these successes, our data proved to be ultimately insufficient to allow us to distinguish between gradual-decay and sudden-death models of short-term visual memory decay.

\section{Experiment}

We conducted a VWM change-detection task involving color stimuli. On each trial, three colors from a 360-degree color wheel were briefly displayed in simultaneous fashion at distinct spatial locations on the computer screen. On the key trials of interest, all three study colors were highly discriminable from one another (adjacent colors from the wheel were 8298 degrees apart). Following a variable retention interval (1, 2, 4 , or $10 \mathrm{~s}$ ), a single test-probe color was presented at one of the spatial locations on the screen. The test probe was either the same as the original color; 16, 32, or 48 degrees away (smallchange trials); or roughly 90 or 180 degrees away (big-change trials). Across blocks, change trials occurred with probability $.3, .5$, or .7. Subjects were informed of the objective change probability at the start of each block and were encouraged to adjust their response biases in accordance with the operating change probability. For example, on blocks in which change probability was high (.7), subjects were informed that if they were guessing, they could achieve more correct answers if they guessed "change." The manipulation of response biases across blocks yields greater constraints for distinguishing alternative models of change-detection performance (Donkin, Nosofsky, Gold, \& Shiffrin, 2013; Rouder et al., 2008).
Two issues that arise in trying to discriminate between sudden-death and gradual-decay explanations of VWM loss involve spatial-position uncertainty and verbal-labeling strategies. When the observer is tested regarding the identity of an object from a given spatial position, there may be some probability that the observer makes the judgment with respect to an item from the wrong position (e.g., Bays et al., 2009; Donkin, Tran, \& Pelley, 2015; Dubé, Zhou, Kahana, \& Sekuler, 2014). One approach to addressing this issue is to conduct paradigms in which the observer is presented with only a single object from a single spatial position on each trial. Unfortunately, this alternative procedure makes it much more likely that observers will generate verbal labels for the to-be-remembered objects. Under such conditions, the memory becomes a complex amalgam of true VWM along with verbal codes, and specialized techniques are needed to tease the components apart (Donkin et al., 2015). Following Zhang and Luck (2009), in the present study observers were presented briefly and simultaneously with three visual objects in order to minimize verbal-labeling strategies. In addition, they were given explicit instructions to avoid using verbal-labeling strategies and to silently repeat the word the if they found themselves using such strategies.

Although we will not be able to rule out the possibility that positional uncertainty contributes to our forgetting data, we believe that our use of three highly discriminable to-beremembered objects together with Far-probe test trials helps reduce the role of such uncertainty. In particular, to the extent that the observer responds "same" on big-change trials, it cannot be because the test probe is similar to some object from the incorrect spatial position: In our design, Far test probes are distant from all three study objects. In addition, on trials with small changes, the test probe will tend to be similar to only the probed study object, making even clearer to the observer which of the to-be-remembered objects is the relevant one.

\section{Method}

\section{Subjects}

The subjects were five members of the Indiana University community who were paid for their participation. Each subject participated for between 9 and 11 sessions, with each session lasting approximately 1.5 hours. Subjects were paid at the rate of $\$ 10$ per hour, with small bonuses for good performance. The subjects all had normal or corrected-to-normal vision, and all reported having normal color vision. None of the subjects was aware of the issues under investigation in the research.

\section{Stimuli}

The stimuli, similar to those described in Zhang and Luck $(2008,2009)$, were 180 colors that were evenly spaced around 
a circle in the $L * a * b *$ color space $(L=50, a=10, b=10$, with a radius of 40 units). The colors were presented as $30 \times$ 30 pixel squares within a $200 \times 200$ pixel region centered on the computer screen. The background color of the screen was white.

All stimuli were generated using MATLAB (version 7.1) and the extensions provided by the Psychophysics Toolbox (Brainard, 1997). The stimuli were presented on a single Apple iMac computer with an integrated Sony Trinitron Multiscan 420GS CRT at a frame rate of $100 \mathrm{~Hz}$ (resolution: $1024 \times 768$ pixels; size: $38.25 \times 28.5 \mathrm{~cm}$ ). The luminance and color calibration measurements were obtained using in-house software and a Photo Research PR-174 SpecraScan radiometer. The maximum and minimum displayable luminances were $131.7 \mathrm{~cd} / \mathrm{m}^{2}$ and $0.02 \mathrm{~cd} / \mathrm{m}^{2}$, respectively. Viewing distance was approximately $57 \mathrm{~cm}$, and the visual angle of the individual squares was approximately $.75^{\circ} \times .75^{\circ}$.

\section{Procedure}

On each trial, three colored squares were presented on the computer screen in random locations within the central rectangular region, subject to the constraint that the centers of each square were at least 60 pixels away. For ease of description, we denote the three squares on the key trials of interest as "left," "middle" and "right" with respect to their status on the color wheel. On each such trial, the middle-color square was selected at random from the color wheel; the left-color square was 82 to 98 degrees less than the middle square (chosen randomly within this interval); and the right-color square was 82 to 98 degrees greater than the middle square. (Left and right values less than 0 or greater than 360 degrees were translated to appropriate values on the 360-degree color wheel.) We used these widely spaced colors on the key trials in order to minimize certain potential effects of positional uncertainty on the change-same judgments (see below). On filler trials, which were presented with probability .2, the three study colors were chosen at random from the color wheel.

On each trial, a single randomly chosen location from the study array was probed with a test square presented at that location. The test probe was either the same color as the original study square; a small-change color $\left(16^{\circ}, 32^{\circ}\right.$, or $48^{\circ}$ away from the relevant study square); or a big-change color $\left(172^{\circ}\right.$ to $188^{\circ}$ from the middle color square). Note that on these bigchange or "Far" trials, the test probe was far from all three study squares, so any confusions on such trials would be unlikely to arise from small gradual losses of precision and/or positional uncertainty (see Modeling Analyses section).

There was a variable retention interval $(1,2,4$, or $10 \mathrm{~s})$ between the presentation of the study colors and the test probe. The retention interval on each trial was chosen at random.

Each session of the experiment was divided into nine blocks of 56 trials each. We manipulated objective change probability across blocks: $.3, .5$, or .7. Each change-probability condition occurred once every three blocks in a random order. Within each block, if a trial was selected to be a change trial, then the degree of change $\left(16^{\circ}, 32^{\circ}, 48^{\circ}\right.$, or "Far") was chosen at random.

All trials began with a $500 \mathrm{~ms}$ fixation asterisk, followed by the presentation of the three study squares $(500 \mathrm{~ms})$. The screen then went blank for the chosen retention interval minus a 200-ms cue time. A 200-ms asterisk cue then appeared at the location of the to-be-presented test probe, which was presented immediately after the cue. The test probe remained on the screen until the subject made a change or same judgment by pressing an appropriate button on the keyboard $(\mathrm{J}=$ change, $\mathrm{F}$ = same). Following the response, text feedback ("CORRECT!" or "INCORRECT") was provided for $1 \mathrm{~s}$ at the center of the screen. Following each block, subjects were informed of their overall percentage of correct responses.

Subjects were informed at the start of each block of the objective change probability operating during that block. They were instructed to adjust their response biases in accord with the objective change probability. Subjects were also instructed to rest their left and right index fingers on the $\mathrm{F}$ and $\mathrm{J}$ keys throughout each block and to press the appropriate key as soon as they made their same versus change judgment. Although we measured subjects' response times (RTs), the RT results were used solely for the purpose of trimming the data and were not the subject of any modeling analyses.

\section{Results}

We deleted from analysis all trials in which the RT was less than $180 \mathrm{~ms}$ or greater than $3,000 \mathrm{~ms}$ (less than $1 \%$ of the data). Such trials were likely cases involving motor-response errors or failures to attend to the task. Each of the individual subjects showed similar patterns of results. Therefore, although we conduct modeling analyses at the level of individual subjects, we present the data averaged across the subjects to illustrate the main trends.

The data are shown at their most fine-grained level in Fig. 2, which plots the probability of change judgments as a joint function objective change probability, probe distance, and the retention interval. (The different symbol types show the observed data, whereas the different line types are the predictions from a full version of the formal model to be presented in the Modeling Analyses section.) To allow for easier observation of the main trends, Fig. 3 plots the results after averaging across the different retention intervals, whereas Fig. 4 plots the results after averaging across the different objective change-probability conditions.

As can be seen in Fig. 2, regardless of the level of objective change probability $(c p)$ and the retention interval, respondchange probabilities were greatest for the Far stimuli, 


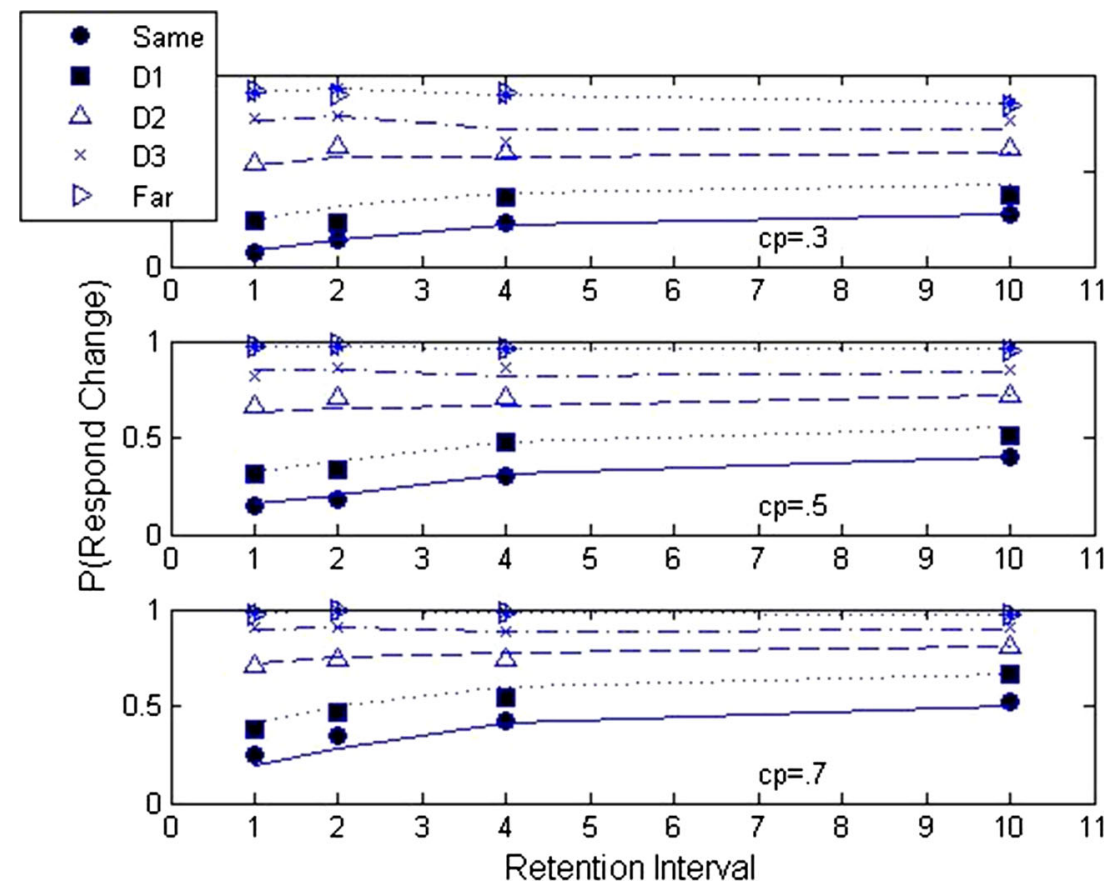

Fig. 2 Mean $\mathrm{P}($ Respond Change) judgments plotted as a joint function of change probability ( $c p$ ), stimulus type (Same, D1, D2, D3 and Far), and the retention interval $(1,2,4$ or 10 seconds). Different symbol types

decreased systematically as the distance of the probe from the to-be-remembered stimulus got smaller (D3, D2, D1), and were lowest when the test probe was the same as the to-beremembered stimulus (see also Figs. 3 and 4). In addition, respond-change probabilities were greatest when objective change-probability was high $(c p=.7)$ and lowest when objective change-probability was low $(c p=.3)-$ see also Fig. 3. The results of greatest interest concern interactive effects of the retention interval and probe distance (see Figs. 2 and 4). Not surprisingly, for same stimuli, respond-change probabilities

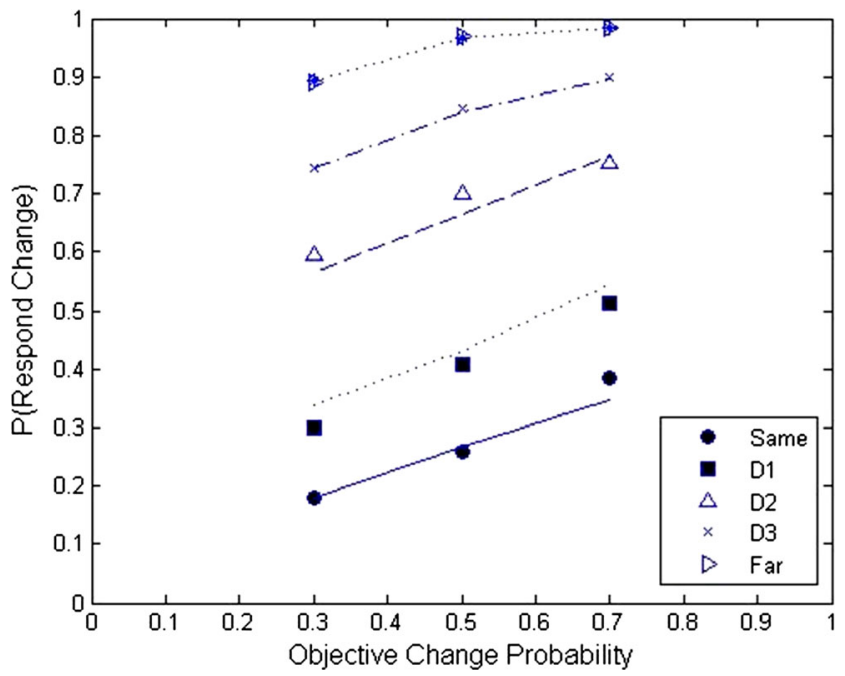

Fig. 3 Mean $P($ Respond Change) judgments plotted as a joint function of stimulus type (Same, D1, D2, D3, and Far) and objective change probability. Symbols: observed data, line types: model predictions represent the observed data and different lines types are the predictions from the full version of a formal model used to analyze the results

(i.e., "false alarms") grew larger as the retention interval increased. The increased false-alarm rate is predicted by essentially all models that assume that forms of forgetting increase with the retention interval. Interestingly, however, respondchange probabilities for stimuli at probe-distance levels D1 and D2 (i.e., "hits") also increased with the retention interval. In other words, at these probe distances, accuracy improved with increases in the retention interval. Finally, at the largest probe distances (D3 and Far), change probabilities either stayed the same or decreased across the retention interval.

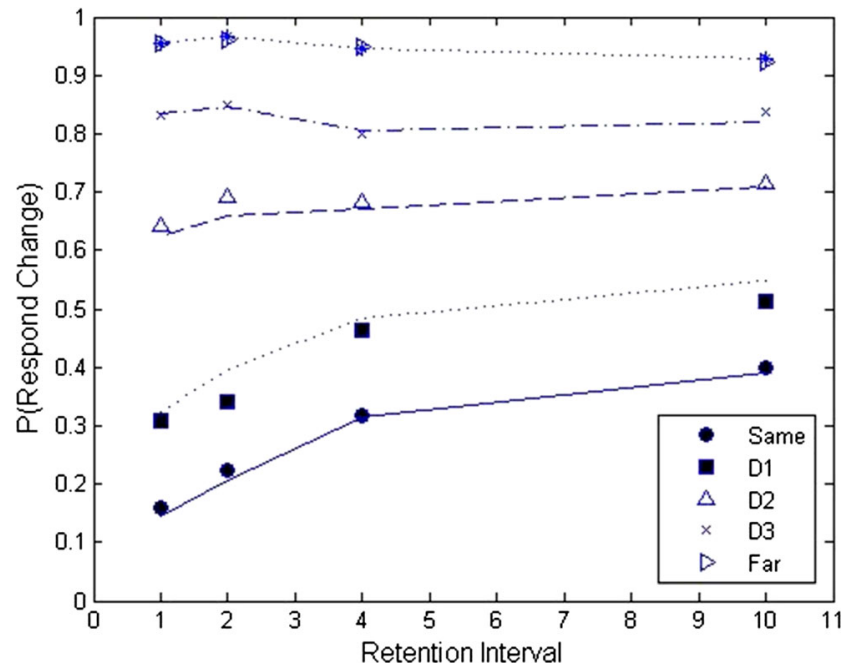

Fig. 4 Mean P(Respond Change) judgments plotted as a joint function of stimulus type (Same, D1, D2, D3 and Far) and the retention interval (seconds). Symbols: observed data, line types: model predictions 
These patterns of results will prove challenging for VWM models based solely on the assumption that only perceptual confusability increases with the retention interval. It is also worth noting here that in the $c p=.3$ condition (top panel of Fig. 2), the probability of change judgments is clearly less than unity for the Far stimuli. Intuitively, this result provides evidence of guessing behavior (i.e., responding based on zero stimulus information), because it is unlikely that the Far probes would ever be perceptually confused with the to-beremembered stimulus. We provide documentation of this point in the Modeling Analyses section.

In Fig. 5 we report the results in terms of $d$ ', defining false alarms as the probability of change judgments to same probes, and hits as the probability of change judgments to change probes. As can be seen, $d$ ' decreases as the distance of the probe to the to-be-remembered stimulus gets smaller and also decreases with the retention interval. These same patterns were observed at all three levels of objective change probability. The $d$ ' results provide an immediate clue that the performance changes across the retention interval involve some form of true memory loss and are not due solely to criterion shifts.

\section{Modeling analyses}

\section{Modeling framework}

The most general models that we use for fitting the data and interpreting the results assume that the change judgments arise from a mixture of two processes: one based on perceptual memory and one based on guessing. If the to-beremembered object has been encoded and has not undergone sudden death, then the observer is presumed to use her

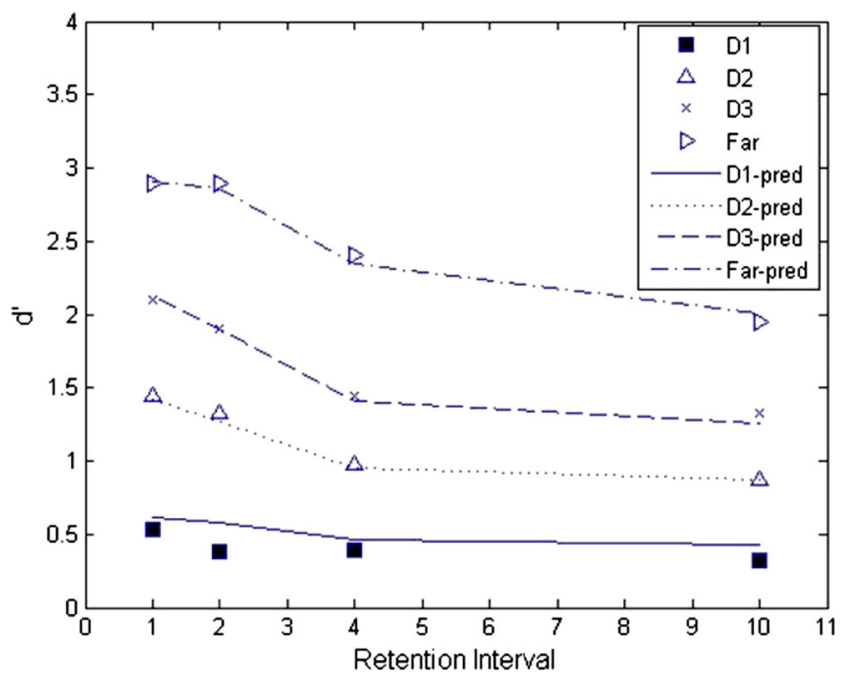

Fig. 5 Mean $d^{\prime}$ values plotted as a joint function of stimulus type (D1, D2, D3, and Far) and the retention interval (seconds). Symbols: observed data, line types: model predictions perceptual memory to make the change-same judgment. Otherwise, the subject must rely on guessing. The probability that the studied object resides in perceptual memory ( time of test is assumed to depend solely on the retention time $t$. Given that it resides in perceptual memory, the probability that use of that memory leads the observer to make a change judgment is denoted $m e m c$ and depends jointly on probe-distance $d$, retention-time $t$, and objective-change-probability $c p$. In the most general version of the model, if the memory for an object has undergone sudden death, then the probability that the observer guesses "change" $(g)$ is allowed to depend jointly on the level of objective change probability $c p$ and on the retention time $t$. Thus, as a general framework for analyzing the data, we assume that the probability that the observer responds "change" given probe-distance $d$, retention-time $t$, and objective-change-probability $c p$ is given by

$$
\begin{aligned}
\mathrm{P}(\mathrm{C} \mid d, t, c p)= & \operatorname{pmem}(t) \cdot \operatorname{memc}(d, t, c p) \\
& +[1-\operatorname{pmem}(t)] \cdot g(c p, t) .
\end{aligned}
$$

We used two approaches to modeling the probability that use of perceptual memory leads to a change judgment (memc). In both approaches, following Shepard (1987), we assumed that the similarity $s$ between the probe and the to-beremembered object was an exponential decay function of their psychological distance $d$,

$s=\exp (-\kappa \cdot d)$,

where $\mathrm{K}$ is a sensitivity parameter. Changes in the $\mathrm{k}$ parameter lead to the changed slopes of the exponential similarity functions depicted in Fig. 1.

In the first approach, which we refer to as the sensitivitybased approach, the psychological distance $d$ between the probe and the to-be-remembered item was given deterministically by the scaling provided by the $\mathrm{L}^{*} \mathrm{a}^{*} \mathrm{~b} *$ color space: $d=$ 0 , if the probe was the same as the item; $d=1,2$, or 3 , for distances D1, D2, and D3, respectively; and $d=7.5$ for the Far probes. ${ }^{1}$ We modeled gradual loss of memory precision in terms of a reduced value of sensitivity $(k)$ with retention time (e.g., Nosofsky, 1987). Thus, separate values of $\kappa$ were estimated for retention times $t=1,2,4$, and 10 .

In the second modeling approach, which we refer to as the variability-based approach, the variability $(\sigma)$ of the study-item memory distributions was assumed to increase with the retention interval. Distances $(d)$ between test items and study items were computed with respect

\footnotetext{
${ }^{1}$ Depending on the to-be-remembered item, the Far probe was on average either 5.625 or 11.25 distance units away. For simplicity, we used the weighted distance 7.5 in the reported modeling-analysis results. Spot checks of individual subject results revealed that the exact choice of Far distance within this range led to minuscule changes in model fits.
} 
to these variable memory distributions and transformed to similarities $(s)$ via Eq. 2 (see Variability-Based Approach section for details). Separate values of $\sigma$ were estimated for each retention time.

For both approaches, the probability that use of perceptual memory led to a change judgment (memc in Eq. 1) was then given by

$\operatorname{memc}(d, t, c p)=k(c p) /\left[k(c p)+M_{t} \cdot s\right]$,

where $k(c p)$ is a criterion parameter that is presumed to vary with objective change probability; and $M_{t}$ is the memorystrength of the trace of the to-be-remembered item. Presumably, if memory strength varies, then it gets weaker as retention time $t$ increases.

Forms of Eq. 3 have been used successfully in past efforts to model perceptual recognition data (e.g., Nosofsky et al., 2011; Nosofsky et al., 2014; Nosofsky \& Zaki, 2003; Shin \& Nosofsky, 1992) and it is useful to consider its underpinnings. The factor $M_{t} \cdot s$ can be viewed as the degree to which the memory trace of the to-be-remembered item is activated by the test probe. As described in our introduction, this memory-trace activation is a joint function of the strength of the trace $\left(M_{t}\right)$ and its similarity to the test probe $(s)$. If the activation is strong, then there is a good deal of evidence that the probe is old - thus, a reduced probability that the observer would make a change judgment. Note that as the distance $(d)$ from the probe to the item increases, similarity $(s)$ decreases (Eq. 2), so the observer is more likely to make a change judgment. Thus, the model naturally predicts increasing change judgments with increases in the distance of the test probe from the to-beremembered item.

Next, consider the predicted effect of increased retention time. According to the first modeling approach, as $t$ increases, sensitivity ( $k$ ) decreases. Thus, there is increased similarity of distinct probes to the to-be-remembered item. Therefore, without any additional mechanisms operating, the perceptualmemory-based model is forced to predict that as retention time increases, there should be a reduction in change judgments for all probes that are distinct from the to-beremembered item. Because change judgments instead often increase with the retention interval (see Fig. 4), this pure version of a perceptual-memory-loss model is unable to account for the data.

One approach to accounting for the results, however, is to make allowance for the idea that the memory strength of the to-be-remembered item $\left(M_{t}\right)$ decreases with retention time (Donkin \& Nosofsky, 2012; Nosofsky et al., 2011; Nosofsky et al., 2014). Because activation of the item trace is a joint function of memory strength and similarity, item activation may therefore decrease with retention time, leading to the increased change judgments seen in the data. As will be seen, instead of assuming continuous decreases in memory strength with time, another approach to predicting the increasing change judgments is to assume all-or-none changes in memory strength (i.e., sudden death).

Recall that according to the second approach to modeling precision loss, the sampled distance $d$ has increasing variability as the retention interval increases. Whether this increasing variability would lead to increasing or decreasing values of similarity ( $s$ in Eq. 3 ) depends jointly on the mean probe distance $\left(\mu_{d}\right)$ and the absolute levels of variability $(\sigma)$ that are involved. We will see, however, that without making allowance for some second factor beyond increases in memory variability, the pure variability-based version of the precisionloss model runs into the same problems as does the sensitivitybased version.

Thus far in our discussion the focus has been on the gradual precision-loss component of the model. Alternatively, memory loss may arise due to sudden death, modeled in Eq. 1 in terms of decreases in the pmem parameter with time $t$. In the case of sudden death, the observer is forced to guess whether or not a change has occurred. In the most general version of the model that we tested, the guess-change probability was given by the product of two guess-change factors, the first related to objective change probability and the second to the retention interval:

$g(c p, t)=g_{1}(c p) \cdot g_{2}(t)$.

Presumably, for example, as objective change-probability $(c p)$ increases, the magnitude of the first guess-change factor will increase.

As currently described, the formal model has a surplus of available free parameters for fitting the data. The full version of the sensitivity-based model has 20 free parameters: 4 all-ornone memory parameters (pmem); 4 sensitivity parameters (K); 3 freely varying memory-strength parameters $\left(M_{i}\right.$; without loss of generality, $\left.M_{1}=1\right) ; 3$ criterion parameters $(k) ; 3$ guess-change parameters related to objective change probability $\left(g_{1}\right)$; and 3 freely varying guess-change parameters related to retention time $\left(g_{2}\right.$; without loss of generality, the maximum $g_{2}$ can be set at 1 ). The full version of the variability-based model has 21 free parameters: the 4 sensitivity parameters are replaced by a single sensitivity parameter and 4 perceptual-memory variability parameters. As explained below, potentially more parsimonious versions of the models arise by constraining some of the free parameters a priori. Tests of these constrained models can be used to provide evidence of whether changes in memory strength operate along with changes in memory variability in the change-detection task; and whether forgetting arises due to gradual loss of visual-memory precision or sudden death. 


\section{Model-fitting approach}

We fitted all models to each individual subject's data by using a maximum-likelihood criterion. Specifically, we conducted computer searches for the free parameters that maximized the likelihood function

$L=\prod_{d, t, c p} \mathrm{~N}_{\mathrm{C}}(d, t, c p) \cdot \mathrm{P}(\mathrm{C} \mid d, t, c p)^{\mathrm{F}(\mathrm{C} \mid d, t, c p)} \cdot[1-\mathrm{P}(\mathrm{C} \mid d, t, c p)]^{\mathrm{F}(\mathrm{S} \mid d, t, c p)}$

where the product is taken across all combinations of the factors probe distance $d$, retention time $t$, and objective change probability $c p ; \mathrm{P}(\mathrm{C} \mid d, t, c p)$ denotes the predicted probability of change judgments at that combination of factors (Eq. 1); $\mathrm{F}(\mathrm{C} \mid d, t, c p)$ is the observed frequency of change judgments; $\mathrm{F}(\mathrm{S} \mid d, t, c p)$ is the observed frequency of same judgments; and $\mathrm{N}_{\mathrm{C}}$ denotes the binomial coefficient of $\mathrm{C}$ change judgments from $\mathrm{N}=\mathrm{F}(\mathrm{C})+\mathrm{F}(\mathrm{S})$ total observations.

The fits of the models were evaluated by transforming the $\log$-likelihood $(\ln L)$ values into both Akaike Information Criterion (AIC) values and Bayesian Information Criterion (BIC) values:

$\mathrm{AIC}=-2 \ln L+2 n_{p}$,

$\mathrm{BIC}=-2 \ln L+\ln (N) \cdot n_{p}$,

where $n_{p}$ is the number of free parameters used by the model and $N$ is the number of observations in the data. The latter term in each criterion is a penalty term for use of free parameters. The model that achieves a smaller AIC or BIC is considered to provide a more parsimonious account of the data. Assuming a large sample size, the BIC places a greater penalty on number of free parameters than does the AIC. Thus, whereas the AIC tends to favor more complex models, the BIC tends to do the reverse.

\section{Modeling results: sensitivity-based approach}

The fits of all models to be described in this section are listed in Table 1. We started by fitting the full version of the sensitivity-based model to the data to confirm that it provided a reasonable organizing framework. The predictions from the full model are illustrated along with the observed data in Figs. 2, 3, 4 and 5. (The predictions of the averaged data are obtained by averaging across the model's predictions of each individual subject's data.) As can be seen, the full model achieves precise quantitative fits of all averaged results.

To evaluate the importance of different components of the model, we fitted restricted (i.e., special-case) versions of the model in which various parameters were constrained a priori. We start by describing cases in which the free parameters were critical to achieving good fits and then move to cases in which more parsimonious fits can be achieved. To provide some understanding of the reason for the resulting fit values, we plot predictions from the special-case models of respond-change probabilities as a function of probe distance and the retention interval (see Fig. 6).

To begin, we fitted a version of the model that assumed no sudden death at any retention times (i.e., values of $p m e m=1$ at all values of $t$ ). (Because there was no sudden death, there was no guessing behavior, so the $g_{1}$ and $g_{2}$ parameters are not used either.) As can be seen in Table 1, even using the BIC statistic (which tends to favor simpler models), this "perfect memory" model yielded dramatically worse fits to the data of all subjects than did the full model. The reason for these poor fits is shown in Fig. 6a. Recall that subjects responded "change" to the Far probes at rates significantly less than unity, particularly at long retention intervals in the $c p=.3$ condition. Without allowing for an absence-of-memory state, the perceptualmemory component of the model needs to estimate extremely low values of sensitivity to try to account for those errors (and still falls short in doing so). These low sensitivity estimates then force the model to greatly under-predict the probability of correct change judgments for probes at distance levels D2 and D3. Thus, apparently, there was at least some proportion of trials in which subjects relied on pure guessing behavior in the absence of any perceptual memory for the study stimuli. ${ }^{2}$

A second special-case model of interest that can be easily rejected is one that assumes no changes in any form of memory strength across the retention interval. Unlike the "perfectmemory" model, in this model the all-or-none pmem parameter is allowed to be less than 1; however, its value is held constant across all retention intervals. In addition, the memory-strength parameters from the continuous visualmemory component of the model (the $M_{\mathrm{t}}$ values in Eq. 3) are held fixed across the retention interval. As reported in Table 1, the AIC fits of this constant memory-strength model are dramatically worse than those of the full model for all five subjects, and the BIC fits are much worse for four of the five subjects. The reason for the extremely poor fits is shown in Fig. 6b. Recall that the respond-change probabilities increased for same probes and for D1 and D2 probes as the retention interval increased. Without allowing for any changes in memory strength, the reduced-sensitivity model predicts decreases in respond-change probabilities, not increases. The present version of the model tries to compensate for this difficulty by assuming increasing guess-change probabilities across the retention interval (the $g_{2}$ parameter in Eq. 4). However, this assumption then forces the model to incorrectly predict increasing respond-change probabilities for the D3 and Far probes, so the model yields very poor fits.

\footnotetext{
${ }^{2}$ Recall also that these big-change same judgments cannot be attributed to spatial-position uncertainty because the Far probes are perceptually distant from the items at all spatial locations.
} 
Table 1 AIC and BIC fits of the sensitivity-based models

\begin{tabular}{|c|c|c|c|c|c|c|}
\hline Sensitivity-based models & 1 & 2 & 3 & 4 & 5 & Sum AIC \\
\hline \multirow[t]{2}{*}{ Full (20) } & 320.3 & 303.5 & 284.4 & 314.8 & 290.3 & \multirow[t]{2}{*}{$1,513.3$} \\
\hline & 448.3 & 429.0 & 410.4 & 440.6 & 416.3 & \\
\hline \multirow[t]{2}{*}{ Perfect memory no guessing (10) } & 401.3 & 383.5 & 388.0 & 443.0 & 487.7 & \multirow[t]{2}{*}{$2,103.5$} \\
\hline & 465.3 & 446.3 & 451.0 & 506.0 & 550.6 & \\
\hline \multirow[t]{2}{*}{ Constant mem. strength and mem. prob. (14) } & 397.7 & 400.3 & 340.9 & 402.7 & 324.2 & \multirow[t]{2}{*}{$1,865.8$} \\
\hline & 487.3 & 488.1 & 429.1 & 490.8 & 412.4 & \\
\hline \multirow[t]{2}{*}{ Constant perceptual confusability (14) } & 313.2 & 309.0 & 285.2 & 311.7 & 290.0 & \multirow[t]{2}{*}{$1,509.1$} \\
\hline & 402.8 & 396.8 & 373.5 & 399.8 & 378.2 & \\
\hline \multirow[t]{2}{*}{ Constant mem. prob. and guessing (14) } & 315.2 & 305.0 & 280.3 & 308.7 & 296.9 & \multirow[t]{2}{*}{$1,506.1$} \\
\hline & 404.8 & 392.8 & 368.5 & 396.8 & 385.1 & \\
\hline
\end{tabular}

Note. Top value in each cell $=$ AIC, bottom value in each cell $=$ BIC. Values in parentheses are the number of free parameters used by each model. Mem. $=$ Memory, Prob. $=$ Probability.

To summarize, using the sensitivity-based approach to modeling precision loss, our interim conclusions are that (a) there is a role of a zero-stimulus-information state (i.e., an "absence of memory" state) in the change-detection judgments and (b) above and beyond possible changes in pairwise discriminability, there are losses in individual-item memory
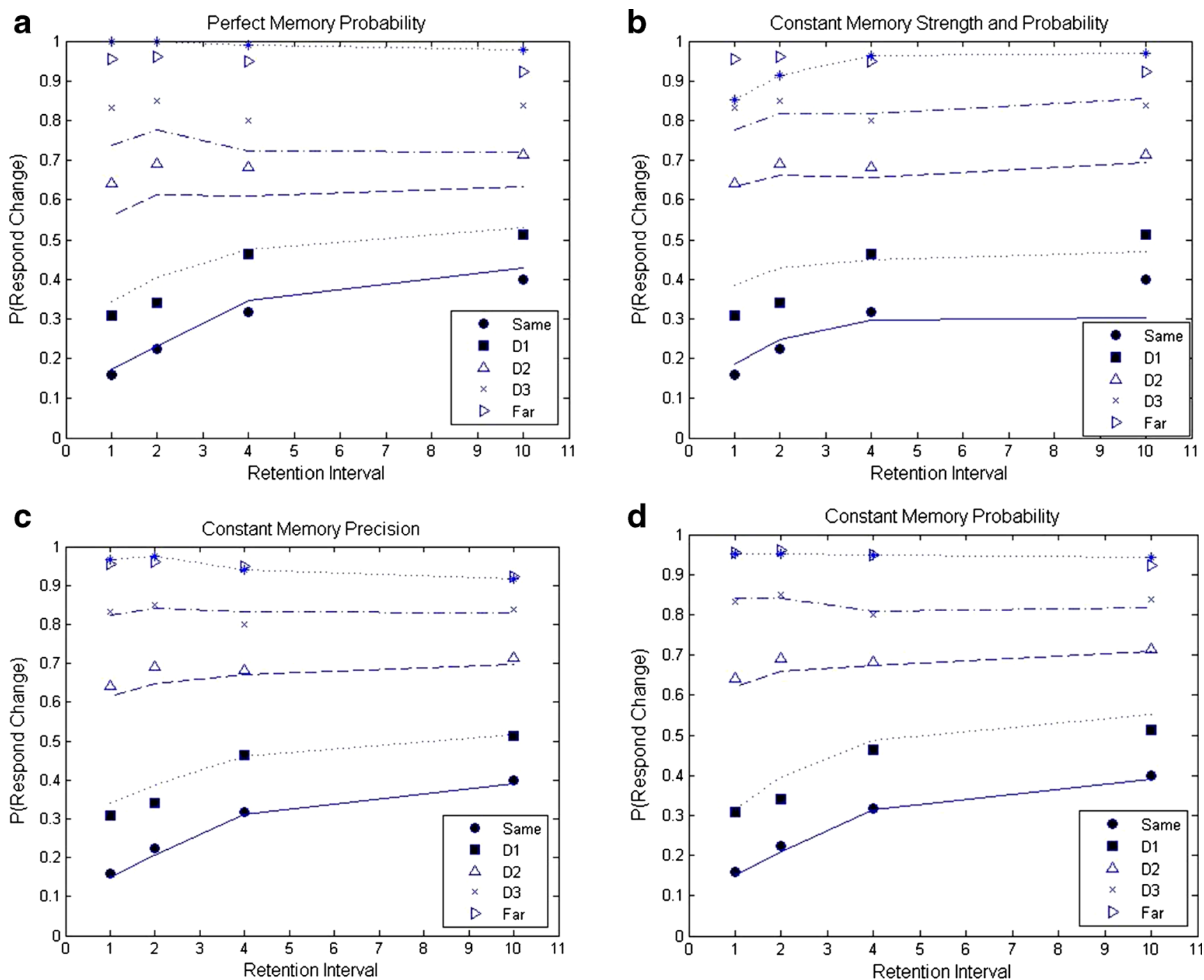

Fig. 6 a. Predictions from perfect-memory-probability model. b. Predictions from constant memory-strength and memory-probability model. c. Predictions from constant memory-precision model. d. Predictions from constant memory-probability model 
strength across the retention interval. However, we have yet to contrast sudden-death versus gradual-decay accounts of the results. For example, the losses in memory strength may be all-or-none (reflecting sudden death) or gradual (the continuous $M_{t}$ parameters). In addition, the model tests thus far do not bear on the question of whether there are gradual changes in memory precision $(\kappa)$ with time.

To address the sudden-death versus gradual-precision-loss hypotheses, we fitted additional special-case models to the data. In a "constant perceptual confusability" model, the continuous memory-strength parameters were held fixed at 1 and the sensitivity parameters were held fixed at a constant value of $\kappa$. Only the all-or-none memory parameters (pmem) and retention-based guessing parameters $\left(g_{2}\right)$ were allowed to vary across the retention interval. As shown in Table 1, the AIC fits of this constant perceptual-confusability model were approximately the same as those of the full model and the BIC fits of the model were considerably better than those of the full model. Thus, the data are consistent with the hypothesis that there were no continuous changes in precision or memory strength across the retention interval and that the results can be interpreted in terms of sudden death and guessing. However, we also fitted a model to the data that assumed a constant probability of using memory (a fixed value of pmem in Eq. 1) and constant guessing across the retention interval $\left(g_{2}\right.$ held fixed at 1). As shown in Table 1, this "constant memory-probability and guessing" model yielded AIC and BIC fits that were very close to those of the constantperceptual-confusability model. Figure $6 \mathrm{c}$ and $\mathrm{d}$ reveal that both special-case models are capable of providing good accounts of how the change-detection judgments varied with stimulus distance and the retention interval. It appears that the present data are not sufficiently strong to sharply discriminate between the sudden-death and gradual precision-loss views.

The best-fitting parameters from these two parsimonious models, averaged across subjects, are reported in Table 2 . The best-fitting parameter estimates are easily interpretable. For the model that assumes constant sensitivity $(\mathrm{K})$ and memory strength $\left(M_{t}\right)$ across the retention interval, the following patterns are observed: First, the memory-storage probabilities (pmem) decrease systematically across the retention interval. Second, the criterion parameters $(k)$ as well as the guessing factor $g_{l}$ increase systematically as objective changeprobability increases. This pattern reflects that the observers show a greater bias to respond "change" as objective changeprobability increases. Finally, the $g_{2}$ estimates indicate that in cases in which observers are in the no-information state, the tendency to guess "change" increases as the retention interval increases. The parameter estimates for the model version that assumes constant memory-storage probability (pmem) and constant $g_{2}$ guessing values are also highly systematic: First, there is a systematic decrease in sensitivity $(\mathrm{K})$ across the retention interval. Second, the continuous memory strengths $\left(M_{t}\right)$ decrease in regular fashion across the retention interval. ${ }^{3}$ And third, the criterion parameters $(k)$ and $g_{1}$ estimates increase as objective change-probability increases.

\section{Modeling results: variability-based approach}

Recall that the second set of models formalized decreasing precision across the retention interval in terms of increased variance of the memory representations rather than in terms of decreased settings of the sensitivity parameter. We implemented versions of the variance-based models that were analogous to those discussed previously for the sensitivity-based approach. We fitted this class of models by using computersimulation techniques. On any given trial, a to-beremembered study item had reference value zero, and its simulated location $h$ at retention time $t$ was a random sample from a standard normal distribution with standard deviation $\sigma_{t}$. The "momentary distance" $d$ associated with a test probe that was $D$ units from the study item was then given by $d=\left|\mu_{D}-h\right|$. This momentary distance would then be transformed to a similarity $s$ via Eq. 2 (cf. Ennis, Palen, \& Mullen, 1988) and prediction probabilities then generated using the same equations as in the sensitivity-based model. The predictions were obtained by using 10,000 simulations at each individual combination of the factors $d, t$, and $c p$.

The resulting fit values are reported in Table 3 . In brief, the pattern of model fits paralleled in most respects the results that we have already discussed for the sensitivity-based approach. The versions of the models that did not make allowance for a zero-information state or that did not make allowance for any decreases in memory strength with the retention interval generally provided poor AIC fits to the data (and sometimes even produced poor BIC fits). By comparison, parsimonious accounts of the data are again available using the two alternative modeling approaches. First, the model that assumes no changes in visual-memory confusability but in which there is sudden death across the retention interval fits well. Second, the model that assumes no increases in sudden death but gradual decreases in visual-memory discriminability (due to increased variance) also fits well. The best-fitting parameter estimates for those two models, averaged across subjects, are reported in Table 4. Again, the patterns of parameter estimates are easily interpretable. According to the model that assumes constant standard deviations $\left(\sigma_{t}\right)$ and constant memory strengths $\left(M_{t}\right)$, it is the memory-storage probabilities (pmem) that decrease systematically with the retention interval. As expected, the criterion parameters $(k)$ and $g_{1}$ estimates increase with

\footnotetext{
${ }^{3}$ In line with related work involving short-term memory search (e.g., Donkin \& Nosofsky, 2012), the memory-strength parameters are almost a perfect power function of the retention interval. A similar relation is observed for the sensitivity-parameter estimates.
} 
Table 2 Mean values of best-fitting parameters for the sensitivitybased models

\begin{tabular}{|c|c|c|}
\hline \multicolumn{3}{|l|}{ Model } \\
\hline Parameter & Constant K, $M_{t}$ & Constant pmem, $g_{2}$ \\
\hline$\kappa_{1}$ & 1.278 & 1.573 \\
\hline $\mathrm{K}_{2}$ & - & 1.296 \\
\hline $\mathrm{K}_{4}$ & - & 0.920 \\
\hline $\mathrm{K}_{10}$ & - & 0.813 \\
\hline$M_{1}$ & {$[1.000]$} & {$[1.000]$} \\
\hline$M_{2}$ & - & 0.509 \\
\hline$M_{4}$ & - & 0.221 \\
\hline$M_{10}$ & - & 0.149 \\
\hline$k(.3)$ & 0.087 & 0.057 \\
\hline$k(.5)$ & 0.134 & 0.065 \\
\hline$k(.7)$ & 0.231 & 0.108 \\
\hline$g 1(.3)$ & 0.627 & 0.257 \\
\hline$g 1(.5)$ & 0.921 & 0.791 \\
\hline$g 1(.7)$ & 0.998 & 0.916 \\
\hline$g 2(1)$ & 0.559 & - \\
\hline$g 2(2)$ & 0.757 & - \\
\hline$g 2(4)$ & 0.932 & - \\
\hline$g 2(10)$ & 0.942 & - \\
\hline $\operatorname{pmem}(1)$ & 0.937 & 0.864 \\
\hline pmem(2) & 0.882 & - \\
\hline pmem(4) & 0.723 & - \\
\hline pmem(10) & 0.606 & - \\
\hline
\end{tabular}

Note. Values in brackets are held fixed a priori. Empty cells indicate cases in which a parameter is either not used in the model or is held fixed at its initial value.

increases in objective change probability. Alternatively, according to the version of the model that assumes a constant memory probability (pmem) and constant guessing across the retention interval $\left(g_{2}\right)$, we see the following: First, the standard deviation of the memory distributions $\left(\sigma_{t}\right)$ increases systematically across the retention interval. Second, the memory strengths $\left(M_{t}\right)$ decrease systematically across the retention interval. And third, the criterion parameters and $g_{l}$ estimates increase as objective change-probability increases. In a nutshell, both the sudden-death and gradual-precisionloss models provide viable and easily interpretable accounts of the data.

\section{Preliminary investigation of variable-resources models}

Our applications of the variability-based models motivate preliminary investigation of another fundamental issue, namely whether a pure variable-resources model can provide adequate fits to the data without requiring the assumption of a zero-information stimulus state that forces guessing. As explained previously, according to variable-resources models, the variability of the memory representation may itself vary considerably across items. If minimal resources are devoted to an item, then the remembered value of that item may be quite distant from the true value.

In our view, however, even if one makes allowance for the possibility of a remembered value that is highly distant from the original, it is unclear how such variable-resources models could account for performance in the present change-detection task without making allowance for a guessing state. The key challenge, in our view, involves cases in which observers fail to respond "change" on big-change (i.e., Far-probe) trials. The issue is illustrated schematically in Fig. 7. The to-beremembered item has reference value zero and the bigchange probe is illustrated far to the right. We illustrate the possibility of a highly dispersed memory representation for the original item in terms of a normal distribution with extreme variability. However, in order for the observer to respond "same" on such a trial, it still must be the case that the particular sampled value from the extreme-variability memory distribution just happens to fall (by "luck") near the bigchange test probe. The likelihood of such an occurrence seems extremely small. Thus, without major adjustment of other parameters (which could impact the model's ability to fit other aspects of the data), the variable-resources model may have difficulty accounting for cases in which the probability of

Table 3 AIC and BIC fits of the variability-based models

\begin{tabular}{|c|c|c|c|c|c|c|}
\hline Variability-based models & 1 & 2 & 3 & 4 & 5 & Sum AIC \\
\hline \multirow[t]{2}{*}{ Full (21) } & 308.0 & 310.2 & 283.0 & 311.1 & 291.6 & \multirow[t]{2}{*}{$1,503.9$} \\
\hline & 442.4 & 442.0 & 415.3 & 443.2 & 423.8 & \\
\hline \multirow[t]{2}{*}{ Perfect memory no guessing (11) } & 346.1 & 374.3 & 374.9 & 383.7 & 394.6 & \multirow[t]{2}{*}{$1,873.6$} \\
\hline & 416.5 & 443.4 & 444.2 & 452.9 & 463.9 & \\
\hline \multirow[t]{2}{*}{ Constant mem. strength and mem. prob. (15) } & 328.6 & 327.7 & 276.4 & 322.6 & 301.1 & \multirow[t]{2}{*}{$1,556.4$} \\
\hline & 424.5 & 421.8 & 370.9 & 417.0 & 395.5 & \\
\hline \multirow[t]{2}{*}{ Constant perceptual confusability (15) } & 304.3 & 311.0 & 286.8 & 311.4 & 290.3 & \multirow[t]{2}{*}{$1,503.8$} \\
\hline & 400.2 & 405.1 & 381.3 & 405.8 & 384.8 & \\
\hline \multirow[t]{2}{*}{ Constant mem. prob. and guessing (15) } & 305.1 & 317.7 & 279.2 & 306.1 & 299.1 & \multirow[t]{2}{*}{$1,507.2$} \\
\hline & 401.1 & 411.8 & 373.7 & 400.5 & 393.5 & \\
\hline
\end{tabular}


Table 4 Mean Values of best-fitting parameters for the variabilitybased models

\begin{tabular}{|c|c|c|}
\hline \multicolumn{3}{|l|}{ Model } \\
\hline Parameter & Constant $\sigma, M_{t}$ & Constant pmem, $g_{2}$ \\
\hline K & 1.726 & 1.602 \\
\hline$\sigma_{1}$ & 0.583 & 0.161 \\
\hline$\sigma_{2}$ & - & 0.673 \\
\hline$\sigma_{4}$ & - & 1.427 \\
\hline$\sigma_{10}$ & - & 1.778 \\
\hline$M_{1}$ & {$[1.000]$} & {$[1.000]$} \\
\hline$M_{2}$ & 一 & 0.732 \\
\hline$M_{4}$ & - & 0.465 \\
\hline$M_{10}$ & - & 0.294 \\
\hline$k(.3)$ & 0.048 & 0.054 \\
\hline$k(.5)$ & 0.081 & 0.062 \\
\hline$k(.7)$ & 0.161 & 0.121 \\
\hline$g l(.3)$ & 0.621 & 0.222 \\
\hline$g l(.5)$ & 0.907 & 0.783 \\
\hline$g l(.7)$ & 0.973 & 0.909 \\
\hline$g 2(1)$ & 0.579 & - \\
\hline$g 2(2)$ & 0.784 & - \\
\hline$g 2(4)$ & 0.945 & - \\
\hline$g 2(10)$ & 0.956 & - \\
\hline pmem(1) & 0.933 & 0.862 \\
\hline pmem(2) & 0.877 & - \\
\hline pmem(4) & 0.718 & - \\
\hline pmem(10) & 0.602 & \\
\hline
\end{tabular}

Note. Values in brackets are held fixed a priori. Empty cells indicate cases in which a parameter is either not used in the model or is held fixed at its initial value.

"same" judgments on big-change trials is non-negligible. By contrast, models that make allowance for guessing from a zero-stimulus-information state do not have this difficulty. If there is an absence of memory for the original stimulus rather than a highly variable memory - then the observer may be just as likely to guess "same" as to guess "change."

To pursue this line of argument, we formulated some rudimentary versions of variable-resources models that did not make allowance for a zero-stimulus-information guessing state but that did make allowance for a high-variance memory state. We again defined four "standard" memory-variance parameters, one for each retention interval. However, we also defined a high-variance parameter for cases in which the observer might develop a highly dispersed memory representation for an item. Finally, we defined four high-varianceprobability parameters, one for each retention interval. Each parameter reflected the probability that a high-variance memory representation was associated with an item at each retention interval. As was the case for our previously described variance-based models, to generate predictions, on each simulated trial a momentary distance $d$ would be sampled from the relevant memory distribution. The distance would then be transformed to a similarity (see Eq. 2) and then substituted into Eq. 3 to generate the predicted probability of a change judgment. In a baseline version of the model, the continuous memory-strength parameters from Eq. 3 were held fixed at 1 , whereas in an extended version the memory-strength parameters were allowed to freely vary. As reported in Table 5, these versions of the no-guessing variableresource models provided poor fits to the data, in accord with the intuitions that we developed in Fig. 7. It remains an open question whether elaborated versions of such models might be developed that do allow one to achieve viable accounts of the present data.

\section{General discussion}

\section{Memory strength}

In summary, in the present VWM change-detection task, we provided model-based evidence that there are decreases in forms of memory strength that go beyond possible decreases in visual-memory precision with time. Whereas changes in memorial precision or variability affect the similarity or confusability between pairs of objects, "strength" is a construct pertaining to individual objects. By combining assumptions regarding individual-item strength and pairwise similarity, various versions of the present change-detection models were able to predict how respond-change judgments varied with the retention interval and test-probe distance. In particular, the models predicted in quantitative detail our findings that respond-change probabilities increased with the retention

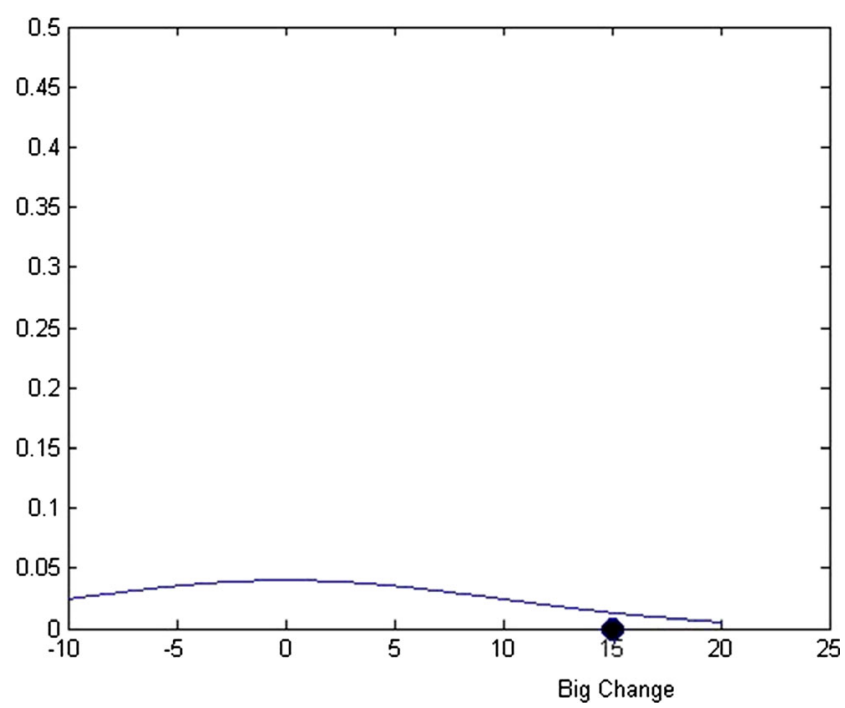

Fig. 7 Schematic illustration of the low probability with which a specific value from a high-variability memory representation will be highly similar to a big-change (Far) test stimulus 
Table 5 AIC and BIC fits of no-guessing variable-precision models

\begin{tabular}{lllllll}
\hline No-guessing variable-precision models & 1 & 2 & 3 & 4 & 5 & Sum AIC \\
\hline Constant memory strength & 343.0 & 340.2 & 322.9 & 361.4 & 374.0 & $1,741.5$ \\
Variable memory strength & 426.2 & 422.4 & 404.8 & 443.2 & 455.8 & 315.7 \\
& 356.5 & 333.9 & 325.1 & 369.8 & $1,701.0$ \\
& 458.9 & 434.3 & 425.9 & 470.5 & 416.4 \\
\hline
\end{tabular}

Note. Top value in each cell $=$ AIC, bottom value in each cell $=$ BIC. Values in parentheses are the number of free parameters used by each model. Mem. $=$ Memory, Prob. $=$ Probability.

interval for "same" probes and for "change" probes that were close in distance to the studied item but stayed roughly constant or decreased for "change" probes that were far from the studied item. Models that did not make allowance for any changes in memory strength (either continuous or all-or-none) failed to account for these findings and provided poor quantitative fits (Tables 1 and 2).

We should acknowledge that, rather than assuming that individual-item memory strength varies, an alternative approach to fitting the change-probability data would be to make allowance for varying response bias across the different retention intervals. One would simply estimate a separate responsebias parameter for responding "change" that grew larger as the retention interval increased. In our view, however, such an explanation is post hoc. The hypothesis that individual-item memory strength decreases with the retention interval is strongly motivated by much past work in cognitive and perceptual psychology, and its translation to the domain of VWM seems compelling and natural.

\section{Zero stimulus-information state versus extreme variability}

A second finding is that adequate quantitative fits to the data relied on the assumption that a zero-stimulus-information state - an absence of memory - operated on some proportion of the trials. On such trials, the observer was forced to guess regarding the test probe's status. This reliance of the good quantitative fits on the zero-information state seemed to primarily reflect the finding that incorrect "same" judgments to bigchange (Far) probes were nonnegligible. A natural interpretation, corroborated by the model fits, is that observers would rarely confuse a study item and a bigchange probe if a memory trace for the study item still existed. Furthermore, because in our design the big-change probes were far in distance from all items in the memory set, the incorrect "same" judgments could not be attributed to location-position uncertainty.

We also conducted preliminary tests of variable-resource models of VWM for the present paradigm. According to those models, the variability of the memory representation may itself vary considerably across trials and across different items from the memory set, in some cases showing extreme variability. However, without making allowance for a zero- information guessing state, the versions of the variableresource models that we tested were also unable to account for the "same" judgments to the Far probes. The key intuition is that although those models make allowance for the possibility that the observer may remember a value for a studied item that is highly distant from the true value of the studied item, the probability that the remembered value happens to be highly similar to any particular big-change probe is still low (see Fig. 7). It remains to be seen if alternative versions of the variable-resources models than we tested here could account for the results.

\section{Limitations and future research}

It is important to remind readers that although plots of the change-probability data suggested that the probability of the zero-information state increased with the retention interval, the data were not strong enough to rule out the possibility that the zero-information state had constant probability (e.g., see Far-probe results in Fig. 6d). Thus, the evidence for the zeroinformation state in the present task could reflect, at least in part, attentional and encoding limitations rather than memory limitations and sudden death. In our view, the most likely possibility is that the zero-information state reflects both encoding and memory failures.

Another limitation of the present study is that we were unable to sharply distinguish between gradual-decay versus sudden-death accounts of the results. Although adequate modeling of the forgetting data required that one make reference to two factors - interitem similarity and a factor that we have termed "memory strength" - the data were not strong enough to allow us to determine whether the decreases in memory strength and precision were gradual and continuous in nature or reflected sudden death. Resolving this fundamental issue remains as a matter for future research.

Third, we examined VWM retention for only a single type of stimulus attribute in the present research, namely color. Although we expect that our findings involving reduced precision and memory strength will be observed across many such attributes, future research is needed to test for the generality of these effects.

Finally, our current account - namely that forgetting from VWM involves a combination of losses in both 
memory precision and memory strength - can be viewed as descriptive in nature. It is important that future work build bridges between our account and those framed at a more mechanistic level, including theories that emphasize varieties of interference (e.g., Endress \& Potter, 2014; Oberauer, Lewandowsky, Farrell, Jarrold, \& Greaves, 2012), failures of consolidation (Vogel, Woodman, \& Luck, 2006), processes of feature matching and feature loss (Shiffrin \& Steyvers, 1997), and interactions among shared neural resources (Swan \& Wyble, 2014). It is an open question whether these more mechanistic theories may capture the types of losses in memory precision and memory strength that appear to underlie the present VWM forgetting phenomena.

Acknowledgments This work was supported by grant FA9550-14-10307 from AFOSR to Robert Nosofsky.

\section{References}

Anderson, J. R. (1990). The adaptive character of thought. Hillsdale, NJ: Erlbaum.

Bays, P. M., Catalao, R. F. G., \& Husain, M. (2009). The precision of visual working memory is set by allocation of a shared resource. Journal of Vision, 9(10), 7.1-711. doi:10.1167/9.10.7

Brady, T. F., Konkle, T., Gill, J., Oliva, A., \& Alvarez, G. A. (2013). Visual long-term memory has the same limit on fidelity as visual working memory. Psychological Science, 24, 981-990. doi:10. 1177/0956797612465439

Brainard, D. H. (1997). The psychophysics toolbox. Spatial Vision, 10, 433-436.

Donkin, C., \& Nosofsky, R. M. (2012). A power-law model of psychological memory strength in short-and long-term recognition. Psychological Science, 23(6), 625-634.

Donkin, C., Nosofsky, R. M., Gold, J. M., \& Shiffrin, R. M. (2013). Discrete-slots models of visual working-memory response times. Psychological Review, 120, 873-902. doi:10.1037/a0034247

Donkin, C., Nosofsky, R., Gold, J., \& Shiffrin, R. (2015). Verbal labeling, gradual decay, and sudden death in visual short-term memory. Psychonomic Bulletin \& Review, 22(1), 170-178.

Donkin, C., Tran, S. C., \& Pelley, M. L. (2015). Location-based errors in change detection: A challenge for the slots model of visual working memory. Memory \& Cognition, 43, 421-431.

Dubé, C., Zhou, F., Kahana, M. J., \& Sekuler, R. (2014). Similarity-based distortion of visual short-term memory is due to perceptual averaging. Vision Research, 96, 8-16.

Endress, A. D., \& Potter, M. C. (2014). Large capacity temporary visual memory. Journal of Experimental Psychology: General, 143(2), 548.

Ennis, D. M., Palen, J. J., \& Mullen, K. (1988). A multidimensional stochastic theory of similarity. Journal of Mathematical Psychology, 32(4), 449-465.

Fougnie, D., Suchow, J. W., \& Alvarez, G. A. (2012). Variability in the quality of visual working memory. Nature Communications, 3, 1229. doi:10.1038/ncomms 2237

Kahana, M. J., \& Sekuler, R. (2002). Recognizing spatial patterns: A noisy exemplar approach. Vision Research, 42(18), 2177-2192.

Kinchla, R. A., \& Smyzer, F. (1967). A diffusion model of perceptual memory. Perception \& Psychophysics, 2, 219-229.

Laming, D., \& Scheiwiller, P. (1985). Retention in perceptual memory: A review of models and data. Perception \& Psychophysics, 37, 189-197.
Luce, R. D. (1963). Detection and recognition. In R. D. Luce, R. R. Bush, \& E. Galanter (Eds.), Handbook of mathematical Psychology (Vol. 1, pp. 103-190). New York: Wiley.

Luck, S. J., \& Vogel, E. K. (1997). The capacity of visual working memory for features and conjunctions. Nature, 390(6657), 279-281.

Magnussen, S., \& Greenlee, M. W. (1999). The psychophysics of perceptual memory. Psychological Research, 62, 81-92.

Nosofsky, R. M. (1987). Attention and learning processes in the identification and categorization of integral stimuli. Journal of Experimental Psychology: Learning, Memory, and Cognition, 13(1), 87.

Nosofsky, R. M. (1991a). Stimulus bias, asymmetric similarity, and classification. Cognitive Psychology, 23(1), 94-140.

Nosofsky, R. M. (1991b). Tests of an exemplar model for relating perceptual classification and recognition memory. Journal of Experimental Psychology: Human Perception and Performance, 17(1), 3.

Nosofsky, R. M., Cox, G. E., Cao, R., \& Shiffrin, R. M. (2014). An exemplar-familiarity model predicts short-term and long-term probe recognition across diverse forms of memory search. Journal of Experimental Psychology: Learning, Memory, and Cognition, 40(6), 1524

Nosofsky, R. M., Little, D. R., Donkin, C., \& Fific, M. (2011). Short-term memory scanning viewed as exemplar-based categorization. Psychological Review, 118(2), 280.

Nosofsky, R. M., \& Zaki, S. R. (2003). A hybrid-similarity exemplar model for predicting distinctiveness effects in perceptual old-new recognition. Journal of Experimental Psychology: Learning, Memory, and Cognition, 29(6), 1194.

Oberauer, K., Lewandowsky, S., Farrell, S., Jarrold, C., \& Greaves, M. (2012). Modeling working memory: An interference model of complex span. Psychonomic Bulletin \& Review, 19(5), 779-819.

Pashler, H. (1988). Familiarity and visual change detection. Perception \& Psychophysics, 44(4), 369-378.

Rouder, J. N., Morey, R. D., Cowan, N., Zwilling, C. E., Morey, C. C., \& Pratte, M. S. (2008). An assessment of fixed-capacity models of visual working memory. Proceedings of the National Academy of Sciences, 105(16), 5975-5979.

Shepard, R. N. (1957). Stimulus and response generalization: A stochastic model relating generalization to distance in psychological space. Psychometrika, 22(4), 325-345.

Shepard, R. N. (1987). Toward a universal law of generalization for psychological science. Science, 237(4820), 1317-1323.

Shiffrin, R. M., \& Steyvers, M. (1997). A model for recognition memory: REM - retrieving effectively from memory. Psychonomic Bulletin \& Review, 4(2), 145-166.

Shin, H. J., \& Nosofsky, R. M. (1992). Similarity-scaling studies of dotpattern classification and recognition. Journal of Experimental Psychology: General, 121(3), 278.

Sims, C. R., Jacobs, R. A., \& Knill, D. C. (2012). An ideal observer analysis of visual working memory. Psychological Review, $119(4), 807$.

Souza, A. S., Rerko, L., Lin, H. Y., \& Oberauer, K. (2014). Focused attention improves working memory: Implications for flexibleresource and discrete-capacity models. Attention, Perception, \& Psychophysics, 76(7), 2080-2102.

Swan, G., \& Wyble, B. (2014). The binding pool: A model of shared neural resources for distinct items in visual working memory. Attention, Perception, \& Psychophysics, 76(7), 2136-2157.

van den Berg, R., Awh, E., \& Ma, W. J. (2014). Factorial comparison of working memory models. Psychological Review, 121(1), 124.

van den Berg, R., Shin, H., Chou, W. C., George, R., \& Ma, W. J. (2012). Variability in encoding precision accounts for visual short-term memory limitations. Proceedings of the National Academy of Sciences, 109, 8780-8785. doi:10.1073/pnas.1117465109 
Vogel, E. K., Woodman, G. F., \& Luck, S. J. (2006). The time course of consolidation in visual working memory. Journal of Experimental Psychology: Human Perception and Performance, 32(6), 1436.

Wilken, P., \& Ma, W. J. (2004). A detection theory account of change detection. Journal of Vision, 4(12), 1120-1135. doi:10.1167/4.12.11
Zhang, W., \& Luck, S. J. (2008). Discrete fixed-resolution representations in visual working memory. Nature, 453, 233-235. doi:10.1038 nature 06860

Zhang, W., \& Luck, S. J. (2009). Sudden death and gradual decay in visual working memory. Psychological Science, 20, 423-428. doi: $10.1111 / j .1467-9280.2009 .02322$ 